\title{
The Dynamics of Equatorially Asymmetric Thermohaline Circulations
}

\author{
JOCHEM MAROTZKE* \\ Center for Global Change Science, Massachusetts Institute of Technology, Cambridge, Massachusetts \\ BARRY A. KLINGER \\ Oceanographic Center, Nova Southeastern University, Dania, Florida
}

(Manuscript received 27 March 1998, in final form 24 May 1999)

\begin{abstract}
The three-dimensional dynamics of equatorially asymmetric thermohaline flow are investigated using an ocean general circulation model in a highly idealized configuration with no wind forcing and nearly fixed surface density. Small asymmetries in surface density lead to strongly asymmetric meridional overturning patterns, with deep water formed in the denser (northern) hemisphere filling the abyss. The poleward deep transport in the lighter hemisphere implies that the deep zonal-mean zonal pressure gradient reverses across the equator. Density along the eastern boundary and the zonally averaged density are nearly symmetric about the equator except at very high latitudes; the Southern Hemisphere western boundary thermocline, in contrast, is balanced by weaker upwelling and is hence broader than its northern counterpart. This pattern is explained through the spinup of the asymmetric circulation from a symmetric one, the timescale of which is set through advection by the mean deep western boundary current.

For the strength of the interhemispheric transport, a lower bound of one-half the one-hemisphere overturning strength is derived theoretically for small finite forcing asymmetries, implying that the symmetric circulation is unlikely to be realized. Under asymmetric surface forcing, enhanced mixing in the denser hemisphere suppresses interhemispheric transport. Conversely, very strong cross-equatorial transport is caused by enhanced mixing in the lighter hemisphere. These results indicate that, once the surface densities determine that North Atlantic Deep Water is the dominant ventilating source, its export rate from the North Atlantic is controlled by mixing and upwelling in the rest of the World Ocean.
\end{abstract}

\section{Introduction}

The thermohaline circulation (THC) differs between oceans and between Northern and Southern hemispheres (Gordon 1986; Macdonald and Wunsch 1996). Previous modeling work on equatorially asymmetric THCs has largely focused on multiple equilibria (e.g., Bryan 1986; Manabe and Stouffer 1988; Marotzke and Willebrand 1991), but much less on how convective activity is dynamically linked to the meridional overturning. Notable exceptions were the studies by Hughes and Weaver (1994) and Rahmstorf (1996), both of which diagnosed relationships between meridional density differences

\footnotetext{
* Current affiliation: School of Ocean and Earth Science, Southampton Oceanography Centre, University of Southampton, Southampton, United Kingdom.
}

Corresponding author address: Prof. Jochem Marotzke, School of Ocean and Earth Science, Southampton Oceanography Centre, University of Southampton, European Way, Southampton SO14 3ZH, United Kingdom.

E-mail: jochem.marotzke@soc.soton.ac.uk (high northern minus high southern latitudes) and overturning strength in their respective general circulation models (GCMs). Previously, we found that very small pole-to-pole surface density differences could set up a markedly asymmetric THC in a GCM (Klinger and Marotzke 1999, KM hereinafter; see also Cox 1989; Marotzke and Willebrand 1991). Fundamentally, the cause lies in the weak abyssal stratification. A $1{ }^{\circ} \mathrm{C}$ temperature difference between high northern and southern latitudes is only $4 \%$ of a typical intrahemispheric difference, but easily represents a vertical spacing of $1-2 \mathrm{~km}$ in the deep ocean. Hence a significant portion of the ocean volume is inaccessible to the "lighter" source since it is filled with the denser water. This answer makes a strong asymmetry in deep ocean ventilation plausible, but it does not at all address the dynamical question of how the force balances are set up. In particular, one must explain how the deep zonal-mean zonal pressure gradient reverses at the equator, needed to ensure geostrophic balance of deep unidirectional transport. The dynamical problem involving both hemispheres is the major focus of this paper.

Asymmetries of the THC about the equator are likely 
to arise not only because of asymmetric buoyancy forcing but also because of the spatial distribution of vertical (or, rather, diapycnal) mixing, which strongly controls the strength of deep-water formation (e.g., Munk 1966). The sensitivity of the THC to mixing has been investigated with GCMs that were confined to a single hemisphere in one basin (e.g., Bryan 1987; Colin de Verdière 1988; Huang and Chou 1994; Marotzke 1997; Zhang et al. 1999) or had vertical mixing independent of horizontal position (Toggweiler and Samuels 1998). However, mixing strength in the ocean might differ between the hemispheres because of variations in roughness of bottom topography (Polzin et al. 1997) or in the energy available for mixing, either from tides (Munk and Wunsch 1998) or wind input (Wunsch 1998). Here, we impose equatorially asymmetric vertical diffusivities in some experiments.

Mixing in the ocean is intensified near bottom or side boundaries (Munk 1966; Wunsch 1970; Armi 1978; Ledwell and Bratkovich 1995; Polzin et al. 1997). We use a simple representation of boundary mixing because in the numerical experiments of Marotzke (1997, M97 hereinafter), the boundary mixing runs showed cleaner scaling behavior than the case with uniform mixing. Otherwise, the integrated properties did not differ much (see section 2), so the theory for the strength and structure of the meridional overturning, developed in M97 within the boundary mixing context, is likely to carry over to the case with uniform mixing. Here, we will use and extend the concepts of M97 to formulate a theory of the equatorially asymmetric THC.

As far as we are aware, this work represents the first investigation of the force balances of the equatorially asymmetric THC and of the consequences of equatorially asymmetric mixing; moreover we present the first formulation of a theory describing these processes. This paper is organized as follows. In section 2, the GCM is briefly described. Section 3 details an asymmetric equilibrium solution of the GCM and how it spins up from a symmetric state. For reasons explained in section 3, we also revisit the general question of THC spinup. Section 4 presents a theory of the asymmetric steady state. Section 5 looks at the effects of differing mixing strengths in the two hemispheres, and section 6 contains a discussion and conclusions.

\section{Model description}

The model used is the Geophysical Fluid Dynamics Laboratory (GFDL) primitive equation GCM (Bryan 1969; Cox 1984). The domain extends from $64^{\circ} \mathrm{S}$ to $64^{\circ} \mathrm{N}$ and is $60^{\circ}$ wide; the bottom is at a constant depth of $4500 \mathrm{~m}$. Horizontal resolution is $3.75^{\circ}$ zonally by $4^{\circ}$ meridionally; in some runs it is doubled to $1.875^{\circ}$ zonally by $2^{\circ}$ meridionally. There are 15 levels in the vertical, varying smoothly in spacing from $50 \mathrm{~m}$ near the surface to $500 \mathrm{~m}$ at depth. The model is forced by restoring boundary conditions on both sea surface tem- perature (SST) and salinity (SSS); the target profiles are independent of longitude and vary like the cosine of latitude, with peak-to-peak amplitudes of $27^{\circ} \mathrm{C}$ and 1.5 psu. The restoring timescale is 30 days, which means that SST and SSS, hence surface density, are very nearly prescribed. In some runs, a linear decrease with latitude of the target SST profile is superimposed, starting from the southern boundary. Surface wind stress is set to zero. Standard horizontal and vertical friction parameterizations are used with coefficients $2.5 \times 10^{5} \mathrm{~m}^{2} \mathrm{~s}^{-1}$ and $10^{-2} \mathrm{~m}^{2} \mathrm{~s}^{-1}$, respectively; horizontal diffusivity is set to $10^{3} \mathrm{~m}^{2} \mathrm{~s}^{-1}$. These parameters are the same also in the high-resolution runs. Static instability is removed by a convective adjustment procedure in which the vertical diffusivity is increased to $1 \mathrm{~m}^{2} \mathrm{~s}^{-1}$; the vertical diffusion term is always evaluated implicitly. Unless noted otherwise, asynchronous integration is used (Bryan 1984), with a temperature and salinity time step of 5 days and a momentum and vorticity time step of 2 hours. We will refer to the model potential temperature as temperature henceforth.

Vertical diffusivity $k_{v}$ is zero, apart from the columns adjacent to the side walls where $k_{v}$ is set to $5 \times 10^{-4}$ $\mathrm{m}^{2} \mathrm{~s}^{-1}$, uniformly with depth. This gives an area average of $0.74 \times 10^{-4} \mathrm{~m}^{2} \mathrm{~s}^{-1}$; in some experiments, $k_{v}$ is set to $2 \times 10^{-4} \mathrm{~m}^{2} \mathrm{~s}^{-1}$ in one hemisphere and to $8 \times 10^{-4}$ $\mathrm{m}^{2} \mathrm{~s}^{-1}$ in the other, keeping the average the same. This crude parameterization of boundary mixing mimics the effect of a sloping lateral boundary, in the vicinity of which tidal mixing, for example, might be very strong. For an extensive discussion of numerical issues involved, see M97. Notice that inclusion of an isopycnal mixing parameterization so far does not appear to alter substantially any of the conclusions drawn from the onehemisphere model (J. Scott 1997, personal communication; A. Weaver 1997, personal communication); neither does a widening of the boundary mixing zone ( $\mathrm{J}$. Scott 1997, personal communication). Notice further that the horizontally averaged vertical diffusivity was calculated incorrectly in M97; it should have been 1.12 $\times 10^{-4} \mathrm{~m}^{2} \mathrm{~s}^{-1}$, not $0.9 \times 10^{-4} \mathrm{~m}^{2} \mathrm{~s}^{-1}$. The maximum THC then is $17.9 \mathrm{~Sv}\left(\mathrm{~Sv} \equiv 10^{6} \mathrm{~m}^{3} \mathrm{~s}^{-1}\right)$ under uniform mixing, rather than the reported $15.7 \mathrm{~Sv}$. The boundary mixing run had 19.2 Sv; integral transports are thus more similar than reported in M97.

\section{Numerical results}

\section{a. Asymmetric equilibrium}

We introduce a surface forcing asymmetry about the equator by lowering the target SST profile by a linear function of latitude, such that there is no reduction at the southern boundary and a $1^{\circ} \mathrm{C}$ reduction at the northern boundary. Starting from an equilibrated run without the SST asymmetry ("symmetric solution"), the model ocean is integrated for about 5500 model years until a new equilibrium is reached, which we will refer to as 
(a) Temperature, zonally averaged



(b) Meridional overturning

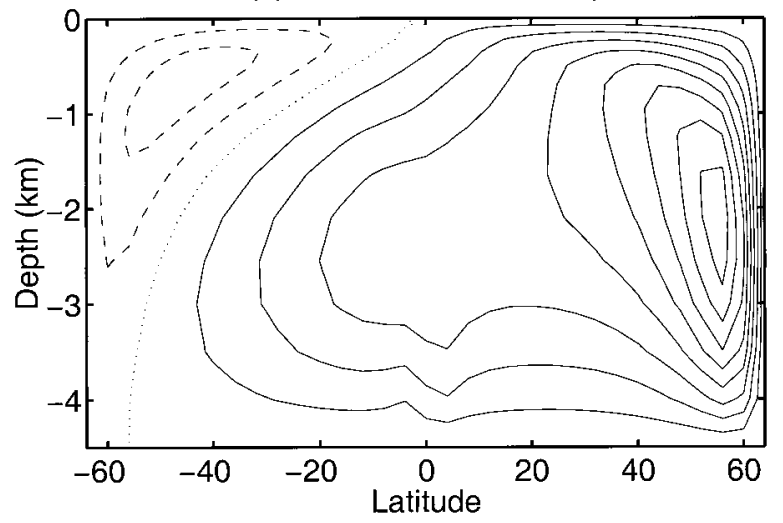

FIG. 1. Standard experiment, steady state: (a) Zonally averaged temperature, contours plotted are $1^{\circ}, 1.5^{\circ}, 2^{\circ}, 3^{\circ}, 4^{\circ}, 6^{\circ}, 8^{\circ}, 12^{\circ}, 16^{\circ}$, and $24^{\circ} \mathrm{C}$. (b) Meridional overturning, contour interval is $2 \mathrm{~Sv}$, the zero contour is dotted, negative values are dashed. The flow is such that high values are to the right, that is, clockwise around a high and anticlockwise around a low.

"standard run." Figure 1 displays the steady-state zonal-mean temperature and the meridional overturning streamfunction. Notice that, because temperature and salinity are forced almost identically and temperature dominates density here, temperature and buoyancy are almost equivalent. While the zonally averaged temperature is very nearly symmetric about the equator, the meridional mass transport shows a strong asymmetry, with 7.7 Sv crossing the equator, a maximum in the north of $17.4 \mathrm{~Sv}$, and a minimum (that is, sinking rate but opposite circulation sense) of $4.8 \mathrm{~Sv}$ in the south. The amplification of small density asymmetries into large overturning asymmetries has been recognized before (Cox 1989; Marotzke and Willebrand 1991; KM); exploring it is one of the main goals of the present work.

The symmetric state from which this integration was started has a THC strength of slightly over $13 \mathrm{~Sv}$ in each hemisphere (not shown), so the sum over both hemispheres is nearly the same in the symmetric and asymmetric solutions, as in KM. We expect the global integral of deep-water formation to be constrained by

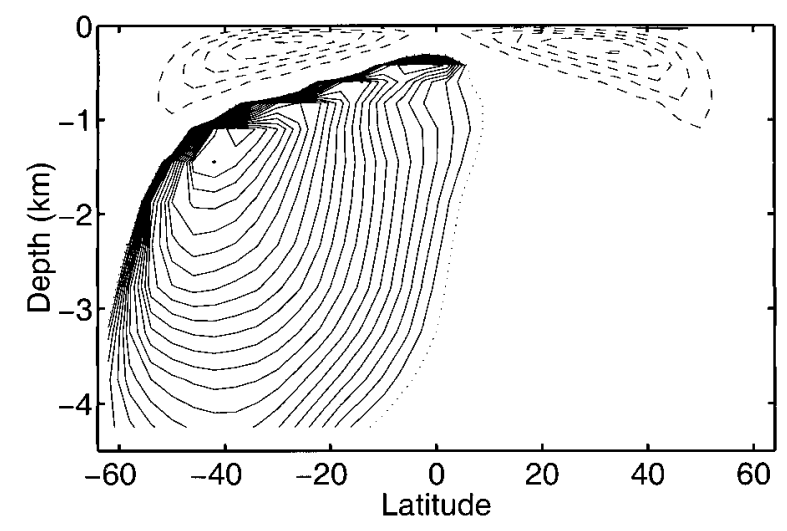

FIG. 2. Standard experiment, steady state: Density difference, eastern boundary minus western boundary; solid: positive values, contour interval is $0.001 \mathrm{~kg} \mathrm{~m}^{-3}$; dashed: negative values, contour interval is $0.1 \mathrm{~kg} \mathrm{~m}^{-3}$. The zero contour is dotted.

the overall vertical diffusive heat flux, which is mainly a function of gross thermocline depth scale and hence similar between the symmetric and asymmetric experiments. The near-constancy of the total deep-water formation rate, independent of the relative strengths of the various THC branches, appears to hold even in global models (Tziperman 1997; Wang et al. 1999), as long as there are no dramatic changes in model geometry such as closing Drake Passage (Cox 1989; Mikolajewicz et al. 1993; England 1993).

To support unidirectional deep meridional transport geostrophically, the deep pressure difference between eastern and western boundaries must change sign at the equator. Figure 2 illustrates how this pressure difference is set up by the density difference between eastern and western walls. Notice that at any given latitude, the eastwest density difference is proportional to the curvature of the meridional overturning, when viewed as a function of depth (see M97). The Northern Hemisphere (NH) exhibits lower densities at the eastern boundary than at the western boundary throughout, except at middepth equatorward of $10^{\circ} \mathrm{N}$. This zonal density difference supports the generally northward shear in the NH. In the Southern Hemisphere ( $\mathrm{SH}$ ), however, eastern boundary density is lower than western boundary density only down to some depth that is about twice the depth of the convection level at the eastern boundary. This can be seen by comparing the depth of the zero line in Fig. 2 to the depth at which isotherms or isopycnals turn from vertical to horizontal in Fig. 3 (below; dashed lines). Notice that twice the convection depth is the depth scale that links vertical shear in overturning to overturning itself (M97). Beneath, the density difference is positive in the $\mathrm{SH}$, albeit smaller by 1-2 orders of magnitude. The northward thermal wind shear accumulates sufficiently over more than $3 \mathrm{~km}$ of vertical extent that a vigorous deep southward transport can be supported.

The subtle but ultimately powerful reversed zonal density difference and the implied hemispheric asym- 


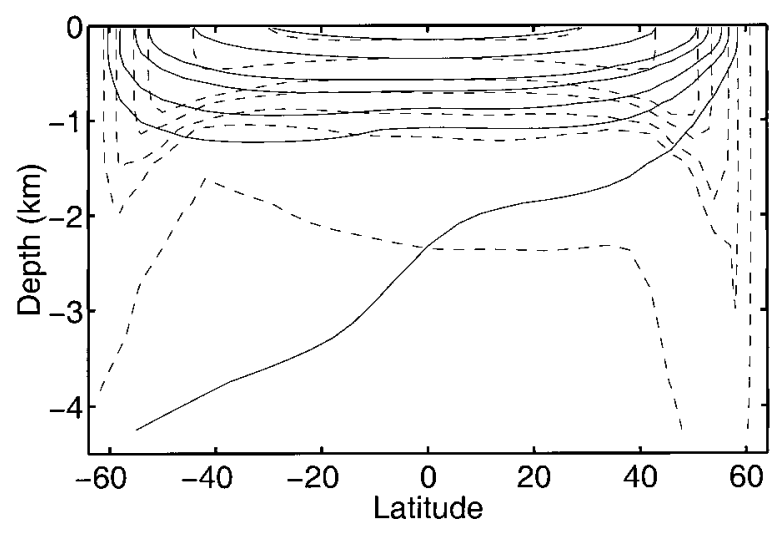

FIG. 3. Standard experiment, steady state: Temperature along the eastern wall (dashed) and western wall (solid); contours plotted are $1^{\circ}, 1.5^{\circ}, 2^{\circ}, 3^{\circ}, 4^{\circ}, 8^{\circ}$, and $16^{\circ} \mathrm{C}$.

metry arise predominantly from the western boundary, as Fig. 3 shows. Apart from the very high latitudes, temperature is very nearly symmetric along the eastern boundary (notice again that temperature and buoyancy are nearly equivalent here, so we plot $\theta$ for convenience). Along the western boundary, however, isotherms drop to a greater depth as one follows them southward across the equator. If the stratification in the vigorous boundary mixing regions is maintained, or at least strongly influenced, by the interplay between vertical upwelling and vertical diffusion, there should be a strong asymmetry in vertical velocity along the western wall as well. Figure 4 shows that the deep thermocline along the $\mathrm{SH}$ western boundary is indeed supported by vigorous downwelling, in contrast to the upwelling in the $\mathrm{NH}$ and one-hemisphere configurations (M97). A comparison of Fig. 4 with vertical velocity at the western wall from the symmetric solution (figure not shown) demonstrates that their difference is nearly antisymmetric about the equator (same magnitude at equal northern and southern latitudes but different signs). Along the eastern boundary of the asymmetric solution, however, the vertical velocity is nearly symmetric equatorward of about $48^{\circ}$ latitude (figure not shown). This near-symmetry helps explain a curious feature of the asymmetric solution: Most of the deep meridional overturning enters the $\mathrm{SH}$ in the deep western boundary current (DWBC, Fig. 5). But, as Fig. 4 shows, there is downwelling in the DWBC area, begging the question of where the necessary upwelling occurs. Figure 5 shows that the deep flow emanating from the DWBC is generally eastward to southeastward, with the relatively warm water to the right, and making up all the flow that reaches the eastern wall. Hence, the entire eastern wall upwelling is fed from the DWBC and thus from the NH. This solution feature is crucial to the dynamics, as will be demonstrated in the theory section.

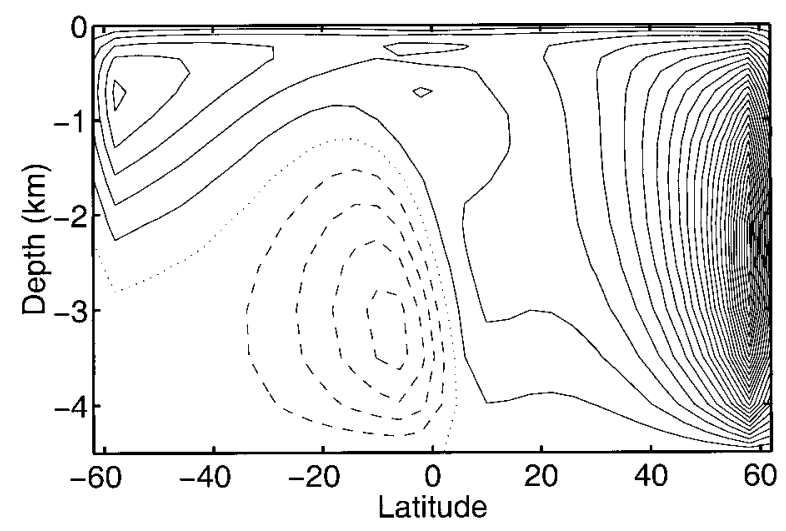

FIG. 4. Standard experiment, steady state: Vertical velocity $w$ along the western boundary, contour interval is $10 \times 10^{-5} \mathrm{~cm} \mathrm{~s}^{-1}$, corresponding roughly to $10 \mathrm{~cm} \mathrm{day}^{-1}$. Areas of downwelling are marked by dashed contours; the zero contour is dotted.

\section{b. Spinup from symmetric flow}

The previous subsection has shown that the asymmetry with respect to the equator arises predominantly along the western wall of the $\mathrm{SH}$, in contrast to the nearly symmetric remainder of the basin. To understand how the model ocean arrives at this relative isolation of the $\mathrm{SH}$ western boundary is the main motivation for now analyzing the spinup from the symmetric solution in some detail. Our results will also show that previously published explanations of the THC spinup must be augmented. In addition, while the spinup of the THC has been investigated before, we are unaware of a paper describing the spinup of purely buoyancy-driven meridional overturning, from symmetric to asymmetric, with the emphasis on the boundary properties as given here. Kawase (1987) considered the response to a prescribed mass injection into the deep (active) layer of a shallow-water model; Suginohara and Fukasawa (1988) the spinup of an asymmetrically buoyancy-forced GCM from rest; McDermott (1996) the time-dependent THC response to changing winds over the Southern Ocean; Döscher et al. (1994) the influence of boundary conditions at the northern edge of their Atlantic model; and Gerdes and Köberle (1995) the short-term (10 years) response of their Atlantic model to changes in Iceland sea surface boundary conditions.

The spinup experiments are performed synchronously (equal time steps for temperature/salinity and momentum/vorticity). Figure 6 shows the time evolution of meridional overturning in the first 90 years after the asymmetric forcing is turned on, by displaying snapshots 9 years apart with the first taken at year 9 (the very early spinup phase of buoyancy-driven flow was investigated by Wajsowicz 1986, and Wajsowicz and Gill 1986, using the GFDL model). At year 9, the asymmetry is barely noticeable; over the next 18 years, sinking in the north intensifies and the upwelling zone of northern source water extends farther southward, but crosses the equator only by a short distance and mainly 
(a)

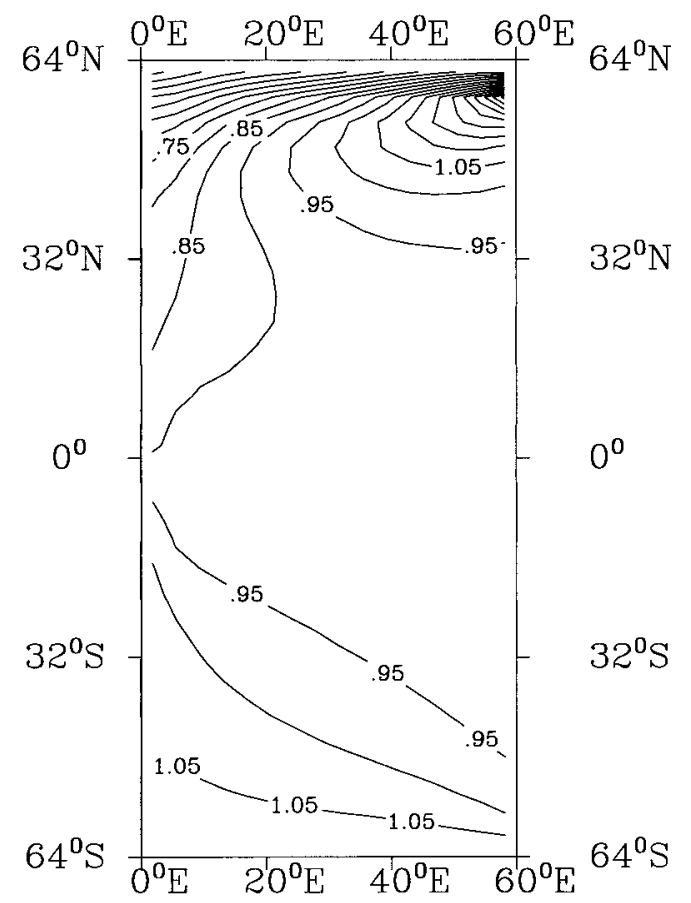

(b)

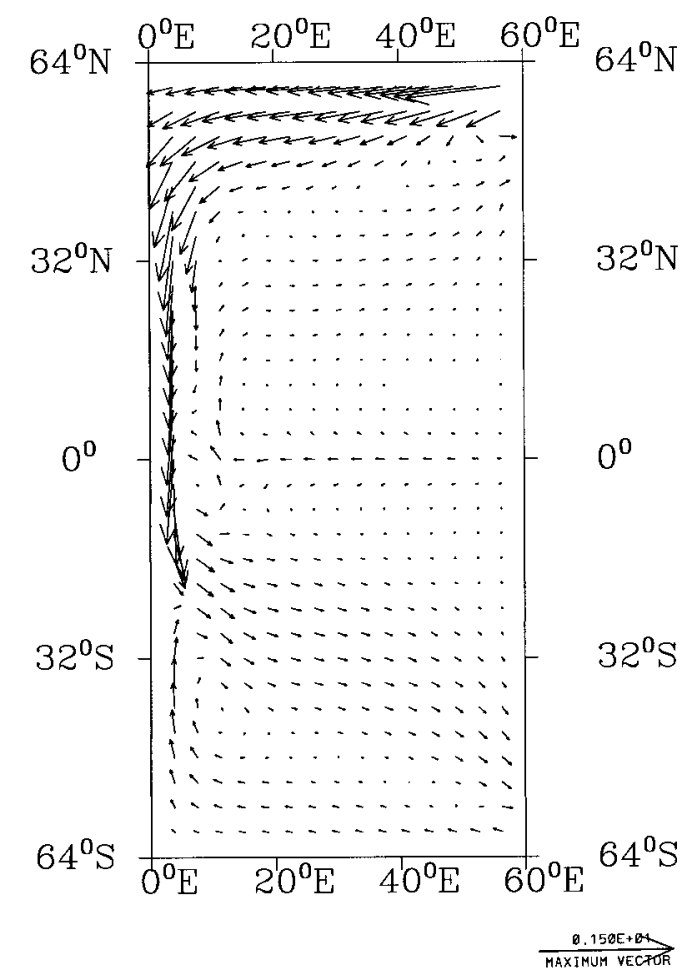

FIG. 5. Standard experiment, steady state, at level 13 (3250 m): (a) Temperature, contour interval $0.05^{\circ} \mathrm{C}$, and (b) horizontal velocity.

in the upper half of the water column. By year 45, the northern overturning has intensified to $26 \mathrm{~Sv}$, near its temporal maximum; notice that the asymmetric steady state has a maximum of only $17 \mathrm{~Sv}$. By year 45, only about $4 \mathrm{~Sv}$ of this northern source water upwell in the $\mathrm{SH}$, within about $10^{\circ}$ of the equator. Otherwise, $\mathrm{SH}$ overturning remains virtually unchanged; indeed, the high-latitude pattern changes very little over the entire 90 years displayed in Fig. 6. At about year 50, a secondary maximum begins to develop at around 3000-m depth, just north of the equator, and very intense upwelling occurs right on the equator. Apparently, the northern overturning cell has stalled in its southward propagation; not until between years 72 and 81 does it invade the $\mathrm{SH}$. The further development is one of farther southward propagation of the northern cell, continuous weakening of the southern cell, and adjustment of the northern maximum to its final value.

To illuminate the evolution of the meridional overturning, Fig. 7 displays the vertically integrated anomalies in vertical velocity, relative to the symmetric state, along the western wall, equator, and eastern wall, and at the same time levels as used in Fig. 6. Increased cooling near the northern wall creates a larger meridional density gradient, which through convection is communicated to great depths and hence causes large zonal thermal wind shear. The increased eastward flow near the surface and westward flow at depth creates increased upwelling near the western wall. These positive anomalies in $w$ (Fig. 7a) and density (not shown) propagate southward and reach the equator after about 45 years. Then, an area of downwelling emerges, which straddles the equator and quickly intensifies; at year 63 it is already quite similar to the steady-state downwelling pattern of Fig. 4.

Figure $7 \mathrm{~b}$ shows again that at year 45 there is deep downwelling near the western wall at the equator; to the east of it, upwelling ensues, which quickly intensifies and propagates eastward. The travel time is about 20 years; by year 72 , the maximum in upwelling has reached the eastern boundary. In its wake, the broad upwelling pattern leaves behind a region of upwelling above downwelling at the western wall and separated at a depth of around $2500 \mathrm{~m}$ (not shown). In the interior, the upwelling decays. Consistent with Fig. 7b, Fig. 7c shows the emergence of the upwelling pattern at the eastern boundary, on the equator, at year 63. Again the pattern intensifies, but now it splits nearly symmetrically, beginning at year 72 . Notice that other processes are going on in Fig. 7c; the increased downwelling in the north is the counterpart to the upwelling in Fig. 7a. The upwelling anomaly in the SH high latitudes is an effect of the reduced convection there and marks a local rather than an interhemispheric process.

From its spatial patterns, the evolution depicted in Figs. 6 and 7 appears consistent with the "standard" picture 

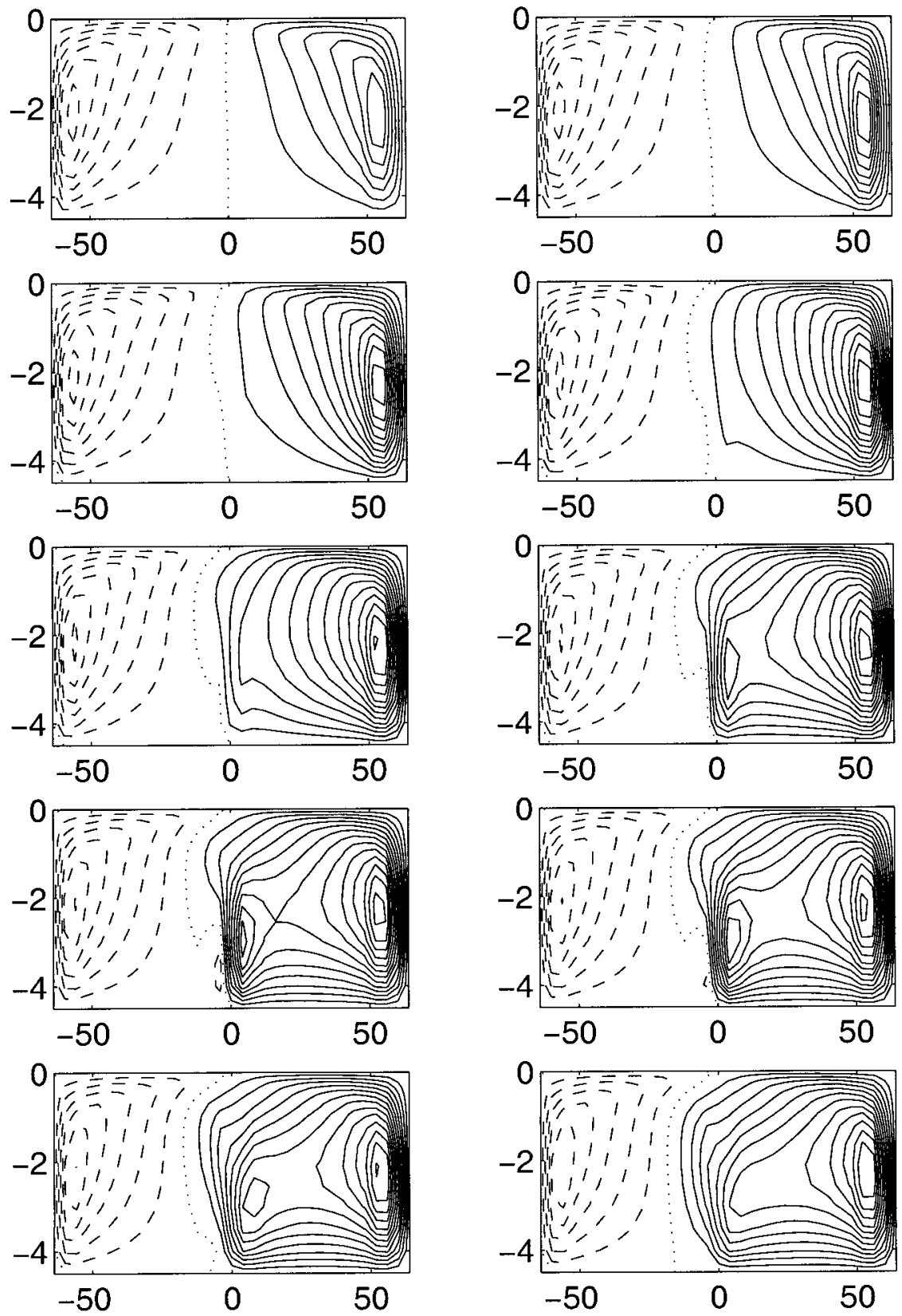

FIG. 6. Spinup toward the standard experiment: Snapshots of meridional overturning, taken 9 years apart, starting at year 9 . The order is first from left to right, then top to bottom. The contour interval is $2 \mathrm{~Sv}$, negative values are dashed, and the zero contour is dotted. The flow is such that high values are to the right, that is, clockwise around a high and anticlockwise around a low.

of the spinup of deep circulation, first described by Kawase (1987). He investigated the response to a mass injection into the northwestern corner of a deep shallowwater layer and found a succession of coastal-equatorialcoastal Kelvin waves, followed by the emanation of long Rossby waves off the eastern boundary. While the presence of Kelvin waves during the spinup has been confirmed repeatedly using GCMs (e.g., Suginohara and Fukasawa 1988; McDermott 1996), the timescale of the evolution in Fig. 7 is inconsistent with the propagation speed of Kelvin waves. Assuming a mean gravity wave speed of $2 \mathrm{~m} \mathrm{~s}^{-1}$, one obtains a travel time down western boundary, equator, and eastern boundary of order 100 days, consistent with Kawase's (1987) numerical results but inconsistent with the 90 year (30000 days) travel time in our experiment. This discrepancy cannot be reconciled despite the numerical effects stemming from the coarse resolution of our model (Hsieh et al. 1983). Ad- 
(a) Western Boundary

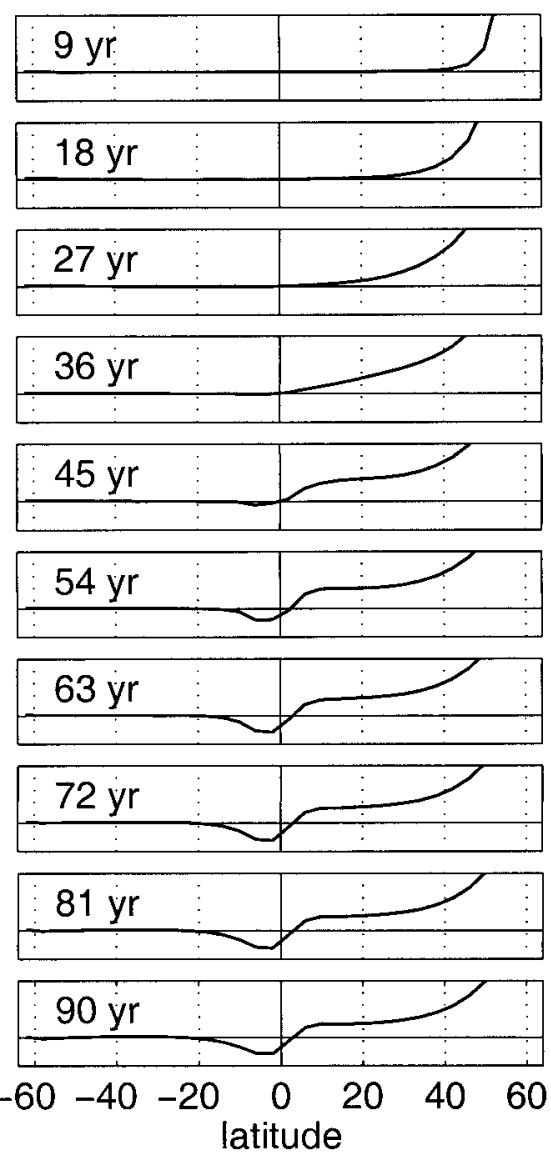

(b) Equator
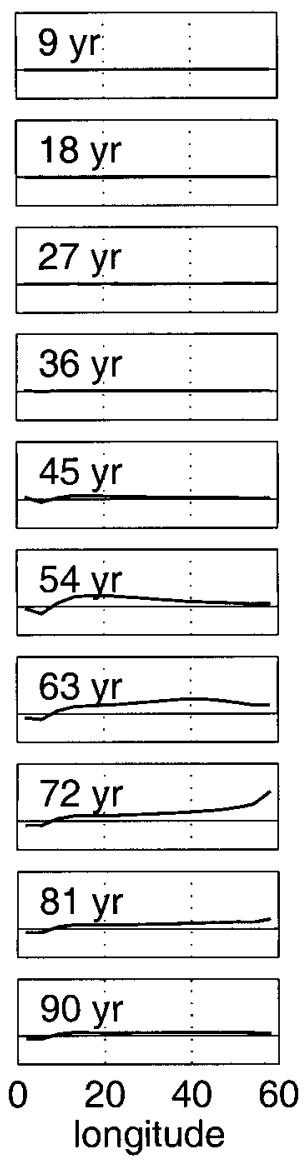

(c) Eastern Boundary
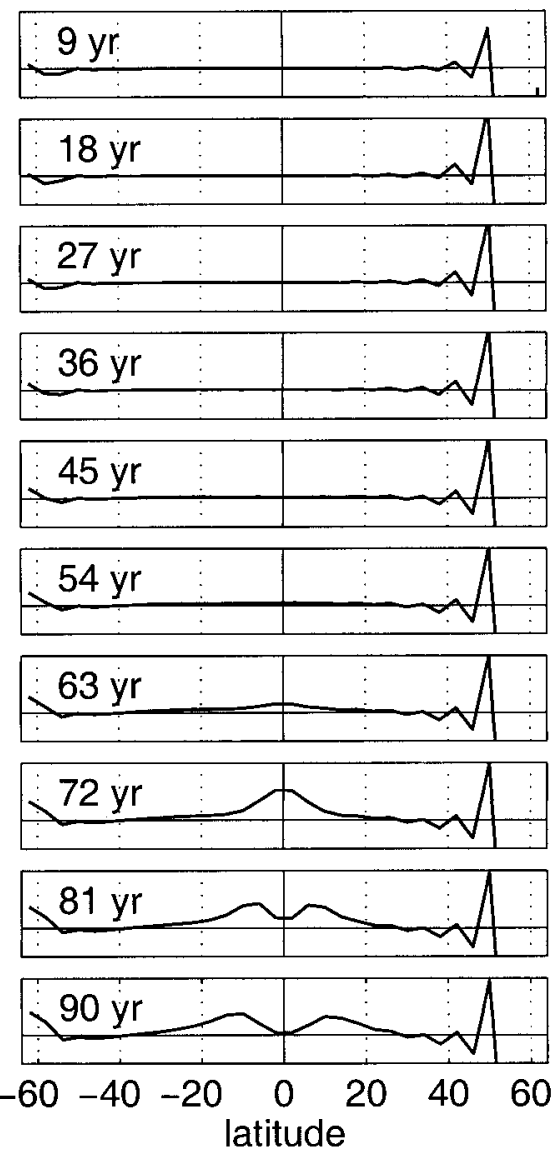

FIG. 7. Spinup toward the standard experiment. Snapshots of the vertically integrated vertical velocity anomaly. Ordinate ranges from -5 $\times 10^{-4} \mathrm{~cm} \mathrm{~s}^{-1}$ to $10 \times 10^{-4} \mathrm{~cm} \mathrm{~s}^{-1}$. (a) Along the western boundary, (b) along the equator, and (c) along the eastern boundary.

justment timescales similar to ours also occurred in the numerical results of Suginohara and Fukasawa (1988; e.g., their Fig. 6a) and McDermott (1996; his Fig. 18d), but the discrepancies with the Kelvin wave travel times were not discussed in these papers.

The spinup is repeated using asynchronous integration (time step of the temperature and salinity equations 60 times that of the momentum and vorticity equations). The results are indistinguishable, whereas Kelvin waves, like all other gravity waves, should propagate more slowly (here by a factor of about 8) during asynchronous integration (Bryan 1984). As a last argument against Kelvin waves as the rate-setting adjustment process in our experiments, notice that Kawase (1987) considered the response to a mass injection into a deep shallowwater layer, whereas we are concerned with the adjustment of the large-scale flow to the water mass changes induced by stronger thermal surface forcing. Convective mixing and deep downwelling are two fundamentally different processes (Marotzke and Scott 1999), and hence plausibly lead to very different dynamical responses.
An explanation of the propagation down the western wall that is consistent with our numerical experiments assumes that the positive density and vertical velocity anomalies, emanating near the northern wall, are simply advected southward by the DWBC. Notice, first, that upwelling and deep positive density anomalies reinforce each other. A dense anomaly at intermediate and great depths, created here by increased zonal and upward flow near the western wall at very high latitudes, causes a high pressure anomaly at depth (below a level of no motion). This leads to enhanced westward flow at the southern flank of the anomaly, upwelling, and further enhanced density. This density anomaly is then advected southward.

To provide a more comprehensive test of whether the DWBC speeds match the travel time along the western wall, we perform two "high-resolution" experiments, each of which comprises a symmetric run followed by the spinup toward the asymmetric steady state. In experiment HI1 horizontal resolution is doubled and all other parameters kept the same as in the "standard experiment" (Figs. 6 and 7); in experiment HI2 the 
resolution and the vertical diffusivity are doubled $\left(k_{v}=\right.$ $10 \times 10^{-4} \mathrm{~m}^{2} \mathrm{~s}^{-1}$ ). Travel time is defined as the time it takes a representative anomaly isopycnal (defined somewhat arbitrarily as $0.01 \mathrm{~kg} \mathrm{~m}^{-3}$ ) to reach the equator. This time is 61 years in HI1 and 45 years in both HI2 and the low-resolution run, which translates into mean speeds of 0.31 and $0.41 \mathrm{~cm} \mathrm{~s}^{-1}$, respectively. This is a reasonable match with the DWBC velocities in the symmetric solutions, which are nearly identical between HI2 and the low-resolution run but lower in HI1. Notice that our sensitivity runs rule out another potential propagation mechanism, the "boundary wave" discussed by Killworth (1985) and Winton (1996). The boundary wave propagates faster in higher resolution, not slower as in HI1 (Killworth 1985); moreover, it crosses an entire hemisphere in one year, as shown by McDermott (1996) in a model of identical resolution to ours (his Fig. 13).

When the deep density anomaly reaches the equator, things change considerably. There is, initially, no crossequatorial DWBC and, because the Coriolis parameter changes sign, deep eastward anomalous flow arises at the anomaly's southern flank, emanating from the western boundary and causing downwelling, hence reduced density. As a consequence, the dense anomaly is arrested right on the equator or, rather, within an equatorial deformation radius. This picture is consistent with the spinup after $\sim 60$ years (Fig. 7a).

The spinup clearly shows that the SH western wall is dynamically isolated from the rest of the basin. We feel that we have found a robust explanation for the propagation of density and upwelling anomalies along the NH western wall. However, we have been unable to devise arguments for the travel times of the propagation along the equator or the eastern walls. Both Kelvin waves and the "boundary waves" are too fast, whereas an image vortex argument at the eastern wall predicts equatorward propagation of a deep dense anomaly (Marotzke and Scott 1999). Further investigation would go beyond the scope of this paper, but is clearly warranted.

\section{Theory}

The overarching goal of this paper is to understand how the east-west density differences are maintained that are in thermal wind balance with equator-crossing meridional overturning. Specifically, this means that we have to ask:

1) Why are eastern boundary stratification and mixed layer depth nearly symmetric about the equator (except at very high latitudes)?

2) Why are thermocline east-west density differences nearly symmetric about the equator?

3) Why are deep east-west density differences strongly asymmetric about the equator, with reversal at or near the equator?
We now develop a theory that results in expressions for the zonal-mean zonal density gradient, as a function of latitude and depth, and for the strength of crossequatorial transport. The starting point is the one-hemisphere theory of M97, which is first briefly recapitulated and then extended to the standard run (asymmetric equilibrium) described in section 3a. Notice that our theory contains a number of empirical elements in that we distill out of the numerical experiments some crucial features, cast these in mathematical terms, and derive their consequences (shown to be self-consistent). Clearly, an entirely deductive approach would be preferable, but we consider the development of a semiempirical theory of the equatorially asymmetric THC significant progress, compared to having no theory at all, which is the current state of the published literature.

\section{a. Recapitulation: One-hemisphere theory (M97)}

In addition to hydrostatic and geostrophic balance, we assume that the surface density, $\rho_{S}(y)$, is given purely as a function of latitude and that the abyss has the properties of the densest surface water. The western boundary region is assumed to have stable exponential stratification, with a constant scale height, $D$, which is part of the solution and arises from vertical advective-diffusion balance. Along the eastern boundary and starting at the equator, isopycnals are assumed flat until they reach their outcrop latitude at which point they turn vertical (upward). The eastern boundary mixed layer depth at any given latitude is hence given by the depth that the outcropping isopycnal has when traced to the equator, which in turn is tied to that at the western boundary at the equator, since in the absence of wind stress no zonal pressure and hence density gradient can be supported along the equator. Through Rossby wave activity, density in the interior is assumed largely to follow that of the eastern boundary, right until the western boundary current.

In one hemisphere, the thermocline scale height $D$ is determined by postulating that upwelling occurs evenly over the eastern and western boundary regions and is balanced by vertical diffusion. One obtains for $D$ and maximum overturning stream function $\Psi_{0}$ the expressions

$$
\begin{aligned}
& D \sim\left(\frac{2 k_{v} \Delta x L_{y}^{2} \beta \rho_{0}}{g\left(\rho_{P}-\rho_{T}\right)}\right)^{1 / 3} \text { and } \\
& \psi_{0} \sim\left[\frac{g}{\beta \rho_{0}} \frac{\rho_{P}-\rho_{T}}{L_{y}}\left(2 k_{v} \Delta x L_{y}\right)^{2}\right]^{1 / 3},
\end{aligned}
$$

where $k_{v}$ is vertical diffusivity, $\Delta x$ zonal grid spacing, $L_{y}$ the latitudinal extent, $\beta$ the variation of the Coriolis parameter with latitude $y, \rho_{0}$ a constant reference density, $g$ gravity, and $\rho_{P}$ and $\rho_{T}$ the surface density at the northern boundary ("polar") and the equator ("tropical"), respectively. Notice that only the product $k_{v} \Delta x$ 
appears but not each factor separately, indicating that $k_{v}$ really is the diffusivity averaged over arbitrary boundary layer width $\Delta x$ (in other words, only the zonal integral over the vigorously mixing region enters). Notice further that Eqs. (1) and (2) are slightly modified compared to M97, where it was assumed that upwelling occurred along the western boundary only. For the same choice of parameters as in the numerical experiments, Eq. (2) gives a maximum overturning of just under 13 $\mathrm{Sv}$, in good agreement with the slightly over $13 \mathrm{~Sv}$ of the symmetric two-hemisphere GCM solution.

\section{b. Two hemispheres: Density along eastern and western walls}

We now include a southern hemisphere having a maximum surface density that is lower than the northern maximum by an amount $\Delta \rho$, which is small compared to $\rho_{P}-\rho_{T}$ (now taken to be the $\mathrm{NH}$ pole-equator density difference). As in M97, we want to relate the overturning transport to the density difference between eastern and western boundaries. Note that merely modifying Eqs. (1) and (2) by the slightly different temperature gradients in the two hemispheres cannot explain the large asymmetries in circulation. We shall show that the "reversed" SH overturning follows from simple but farreaching modifications of the single-hemisphere stratification described above. The key modification is that the SH western boundary thermocline is broader than in the $\mathrm{NH}$ and broader than at the SH eastern boundary, as can be seen in the numerical experiment (Fig. 3). The special, relatively isolated role of the $\mathrm{SH}$ western boundary thermocline arises because the upwelling pattern induced by the surface asymmetry spreads along boundaries and the equator except the SH western boundary, as the spinup experiment shows (Fig. 7). In the following, we will assume that even under asymmetric conditions $\mathrm{NH}$ thermocline thickness and $\mathrm{NH}$ total upwelling are given by Eqs. (1) and (2), respectively. The SH thermocline depths are allowed to differ between eastern and western walls and are denoted $D_{E}$ and $D_{W}$, respectively. Since the following development for the NH would proceed identically to M97, we will only display the equations for the $\mathrm{SH}$.

For simplicity, we initially tried constant $D_{E}$ and $D_{W}$, but this led to large unbalanced zonal density gradients at the equator, in conflict with the GCM (e.g., Fig. 2). The next simplest possibility is $D_{E}$ constant and $D_{W}(y)$ varying smoothly with latitude between $D_{E}$ at the equator and $D_{\max }$ at the southern wall. Following M97, this allows us to express the $\mathrm{SH}$ western boundary density as

$$
\rho_{W}(y, z)=\rho_{P}+\left[\rho_{S}(y)-\rho_{P}\right] \exp \left[z / D_{W}(y)\right]
$$

and the $\mathrm{SH}$ eastern boundary densities as

mixed layer:

$$
\rho_{E}(y, z)=\rho_{S}(y),
$$

below mixed layer:

$$
\rho_{E}(y, z)=\rho_{P}+\left(\rho_{T}-\rho_{P}\right) \exp \left(z / D_{E}\right) .
$$

The mixed layer is defined as $z>z_{\rho}(y)$ (and $z<0$ ), where $z_{\rho}$ is chosen to insure static stability and (simultaneously) continuity of density at the base of the mixed layer (M97). Mixed layer depth $\left|z_{\rho}\right|$ in the SH is related to $D_{E}$ by

$$
z_{\rho}(y)=D_{E} \ln \left[\frac{\rho_{P}-\rho_{S}(y)}{\rho_{P}-\rho_{T}}\right],
$$

where $\rho_{S}(y)$ is surface density (see M97). Equation (4) shows that if $D_{E}$ and $D_{N}$ are not too different, the slightly different surface densities in $\mathrm{NH}$ and $\mathrm{SH}$ lead to mixed layer depths nearly symmetric about the equator, except at high latitudes where $z_{\rho}$ goes to minus infinity (i.e., reaches the ocean floor) only in the $\mathrm{NH}$. In numerical experiments, the maximum mixed layer depth in the $\mathrm{SH}$ is $2100 \mathrm{~m}$ for $\Delta \rho /\left(\rho_{P}-\rho_{T}\right)=0.02$ (section 3) and 950 $\mathrm{m}$ for $\Delta \rho /\left(\rho_{P}-\rho_{T}\right)=0.06$. Equation (4) predicts values of $4 D_{E}$ for the former case and $3 D_{E}$ for the latter $\left(D_{E}\right.$ $\sim 350 \mathrm{~m}$ reasonably well characterizes both experiments). Thus the theory underestimates the sensitivity of mixed layer depth to $\Delta \rho$ but gives the correct tendencies and order-of-magnitude behavior.

Using $D_{W}(y)$ and $D_{E}$ in the $\mathrm{SH}$ western and eastern boundaries, respectively, can produce a qualitative change in the SH zonal density difference, compared to the NH. For any $z$ shallower than $z_{\rho}(y)$, the eastern boundary is lighter than the western boundary, as in the $\mathrm{NH}$ or in M97. However, below the mixed layer, the eastern boundary density approaches its abyssal density more rapidly with depth than does the broader western boundary thermocline. If $D_{E}<D_{W} \ll H$, where $H$ is total ocean depth, the east-west density difference reverses sign (hence is zero) at some finite depth above the bottom. Since the east-west density difference is zero right at the bottom as well, there exists a depth, $z_{\max }$, at which it attains a maximum. The appendix shows [Eq. (A7)] that this maximum is given by

$$
\begin{aligned}
\Delta \rho_{\max }(y) & \equiv \rho_{E}\left(y, z_{\max }\right)-\rho_{W}\left(y, z_{\max }\right) \\
& =\left(\rho_{P}-\rho_{T}\right)\left(\frac{\rho_{P}-\rho_{S}(y)}{\rho_{P}-\rho_{T}}\right)^{1 /(1-d)} d^{d /(1-d)}(1-d),
\end{aligned}
$$

where $d(y) \equiv D_{E} / D_{W}(y)$ is the ratio of the thermocline scale heights in the $\mathrm{SH}$.

Having shown that the above assumptions produce $\mathrm{SH}$ east - west density differneces in qualitative agreement with the GCM (compare Fig. 2), we now test their quantitative agreement by studying $\sigma$, defined as the maximum, with respect to $y$, of $\Delta \rho_{\max }(y)$ in the $\mathrm{SH}$. Assuming that the precise details of the meridional profiles of $d(y)$ and $\rho_{S}(y)$ will not strongly influence the 
(a) Maximum rho ${ }_{e}-$ rho $_{w}$

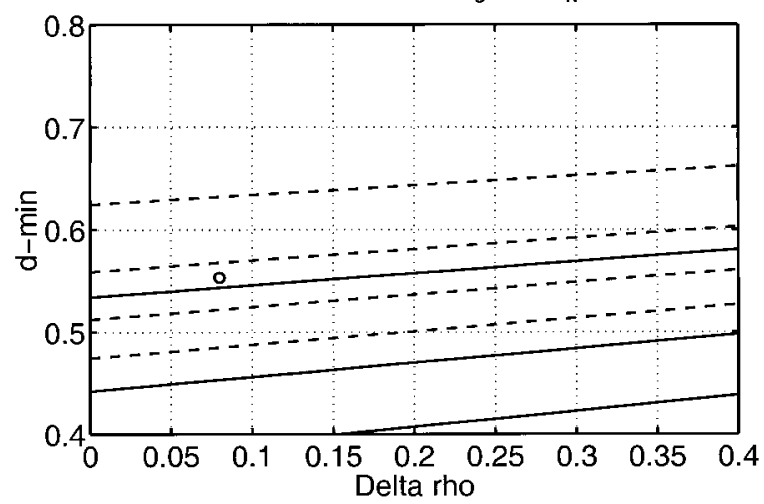

(b) Latitude of maximum rho ${ }_{\mathrm{e}}-\mathrm{rho}_{\mathrm{w}}$

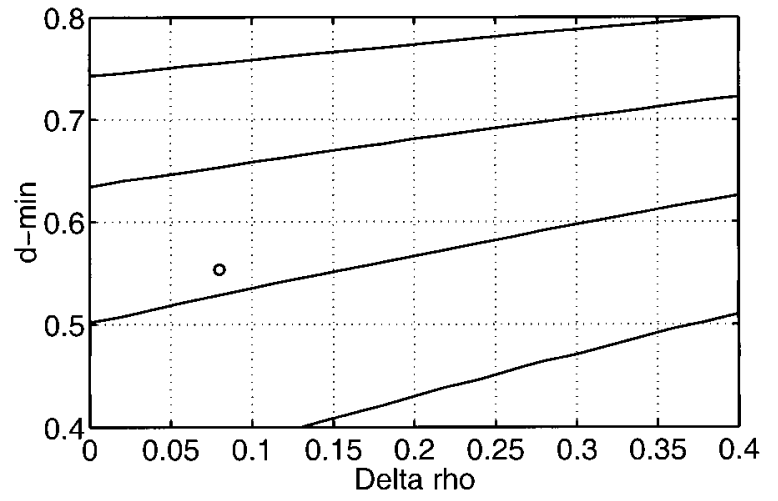

FIG. 8. Theory. All panels have as abscissa the imposed pole-topole surface density difference, $\Delta \rho$, and as ordinate, $d_{\min }$, the minimum ratio of $\mathrm{SH}$ eastern and western scale heights. The circles mark the position of the theoretical solution $\left(d_{\min }=0.5533\right.$ for $\Delta \rho=0.08$ $\mathrm{kg} \mathrm{m}^{-3}$, for the parameter choices listed in the text). (a) Maximum positive subthermocline east-west density difference in the $\mathrm{SH}$, Contour interval is $0.02 \mathrm{~kg} \mathrm{~m}^{-3}$ (solid) or $0.008 \mathrm{~kg} \mathrm{~m}^{-3}$ (dashed, below $0.04 \mathrm{~kg} \mathrm{~m}^{-3}$ only). (b) Latitude where $\sigma$ is attained. Contour levels are $20^{\circ}, 25^{\circ}, 30^{\circ}$, and $35^{\circ} \mathrm{S}$, from top to bottom of the plot.

scaling of $\sigma$, we let both vary linearly with latitude. Hence, $\rho_{S}(y)$ goes from $\rho_{P}-\Delta \rho$ at the southern boundary to $\rho_{T}$ at the equator to $\rho_{P}$ at the northern boundary, and for $\mathrm{SH}$,

$$
d(y)=1+\left(d_{\min }-1\right)|y / L|,
$$

so that $d(y)$ is $d_{\min }$ at the southern boundary and 1 at the equator. Later, we will determine $d_{\text {min }}$ to close the problem, but for now we look at how $\sigma$ depends on $\Delta \rho$ and $d_{\min }$ separately, according to Eq. (5). Figure $8 \mathrm{a}$ shows the very strong dependence of $\sigma$ on $d_{\min }$; for $d_{\text {min }}$ $=0.8, \sigma$ is only about $10^{-4}$ of $\rho_{P}-\rho_{T}$, but $\sigma$ increases by two orders of magnitude as $d_{\min }$ is lowered to 0.4 . In contrast, $\sigma$ is relatively insensitive to $\Delta \rho$. The latitude where $\sigma$ is attained (Fig. 8b) also shows stronger dependence on $d_{\text {min }}$ than on $\Delta \rho$.

A scaling for $\psi_{c}$, the deep overturning in the $\mathrm{SH}$ (interpreted also as the cross-equatorial transport), can now be given as

$$
\psi_{c}=\frac{g}{2 f \rho_{0}} H_{r}^{2} \sigma,
$$

where $H_{r}$ is one-half the depth range over which eastern wall density is higher than western wall density; notice that it is somewhat reduced from but comparable to onehalf the total depth. Here, we will use $H_{r}=2000 \mathrm{~m}$. Owing to the large vertical range over which thermal wind shear can accumulate, a $\sigma$ as small as $0.02 \mathrm{~kg} \mathrm{~m}^{-3}$ (0.005 of $\rho_{P}-\rho_{T}$, as in the GCM, Fig. 2) can support cross-equatorial flow of several Sverdrups. Equations (5), (7), and the definition of $\sigma$ together provide a relationship between $d_{\min }$ and $\psi_{c}$, which we write symbolically as

$$
\psi_{c}=\mathcal{F}\left(d_{\min }\right)
$$

\section{c. Two hemispheres: Advective-diffusive balance for various overturning branches}

We now invoke vertical mixing to find a second relationship between $d_{\min }$ and $\psi_{c}$ [in addition to Eq. (8)], which closes the problem. The argument proceeds in three stages.

1) In the GCM, the interhemispheric mass transport occurs almost exclusively in the DWBC (Fig. 5b) where water downwells (Fig. 4) and turns eastward in thermal wind balance with the deep temperature gradient (warmer water toward the south, Fig. 5a). If the flow fanning out from the DWBC reaches the southeastern corner of the basin, as it does in Fig. 5b, the entire upwelling along the eastern wall is ultimately fed from the NH. We now postulate that this always holds. If, as is plausible, there is a balance between vertical diffusion and upwelling in all side boundary layers, $D_{E} \propto 1 / \psi_{c}$.

2) A representative value of $\mathrm{SH}$ western boundary thermocline scale height is denoted $\left\langle D_{W}\right\rangle$ and is assumed to be determined by a balance between vertical diffusion and upwelling of the southern-source overturning cell, of strength $\psi_{S}$. Hence, $\left\langle D_{W}\right\rangle \propto 1 / \psi_{S}$. If the upwelling areas are the same along the eastern and western boundaries, we obtain for the ratio of thermocline scale heights

$$
\langle d\rangle \equiv \frac{D_{E}}{\left\langle D_{W}\right\rangle}=\frac{\psi_{S}}{\psi_{c}} .
$$

Notice that $\langle d\rangle<1$ since $D_{E}<\left\langle D_{W}\right\rangle$, which in turn is necessary to support the deep transport geostrophically. It follows that $\psi_{c}>\psi_{s}$, to which we will return shortly.

3) The total amount of upwelling in each hemisphere remains similar to the symmetric solution (10-13 Sv, depending on depth horizon; see Fig. 1b) because the gross thermocline structure in either $\mathrm{NH}$ or $\mathrm{SH}$ does not change much during the transition from the symmetric to the asymmetric circulation. The symmetric THC can, in principle, be obtained from the one-hemisphere theory [eq. (2) above] and is assumed known. Hence, we have the constraints on $\psi_{S}, \psi_{c}$, and, by extension, maximum NH overturning $\psi_{N}$, 


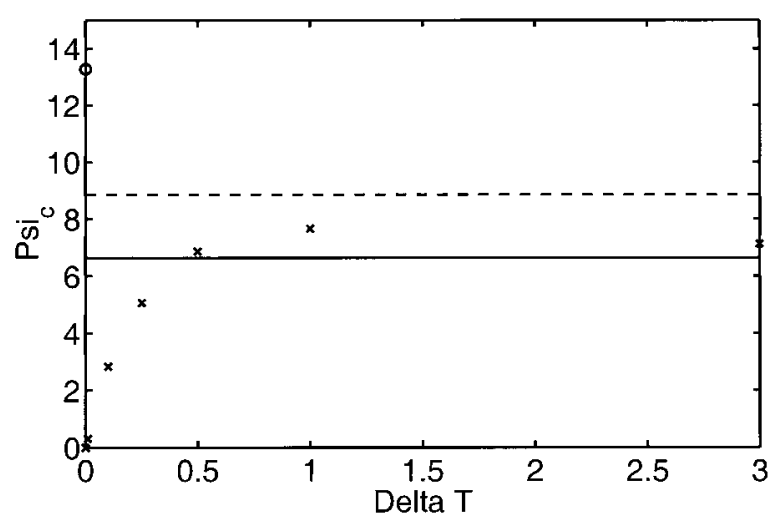

FIG. 9. GCM, steady-state cross-equatorial THC, $\psi_{c}$, as a function of the imposed SST asymmetry (crosses). Circle: symmetric THC strength. Solid and dashed lines mark theoretical lower and upper bound on $\psi_{c}$, respectively.

$$
\begin{aligned}
& \psi_{S}+\psi_{c}=\psi_{0}, \\
& \psi_{N}-\psi_{c}=\psi_{0} .
\end{aligned}
$$

From Eqs. (9) and (10a), $\psi_{S}$ is eliminated to yield

$$
\psi_{c}=\frac{\psi_{0}}{1+\langle d\rangle} .
$$

Since $\langle d\rangle$ depends on $d_{\min }$, Eq. (11) is the desired second relationship between $\psi_{c}$ and $d_{\min }$. From (11) alone and $\langle d\rangle<1$, it follows that

$$
\psi_{c}>\frac{1}{2} \psi_{0}
$$

which expresses the remarkable result that there exists a lower bound on the strength of the interhemispheric transport: If there is any asymmetry at all in the system, at least half as much water must cross the equator as an isolated $\mathrm{SH}$ would have in overturning strength. This conclusion is surprising enough that it justifies a careful examination of exactly what assumptions led to it. Most importantly, no particular shape of $D_{W}(y)$ is required, but merely that $D_{W}(y)>D_{E}$ : Thermal wind balance for deep southward transport requires the western boundary to be less dense than the eastern boundary; that is, there is weaker stratification and hence smaller diffusive flux at the western than at the eastern boundary. The northern-source DWBC water, after its zonal migration across the basin (Fig. 5), balances the relatively stronger downward diffusion near the eastern boundary. Hence, more deep water must enter from the north than is formed in the south, in accordance with Eq. (12) and the assumption that total upwelling in the $\mathrm{SH}$ is a constant. Clearly, the lower bound on $\psi_{c}$ applies only if indeed all upwelling at the $\mathrm{SH}$ eastern wall is fed from northern source water, or, equivalently, the flow emanating from the DWBC (Fig. 5b) reaches the southeastern corner (see below).

Equation (11) also implies the existence of an upper bound on $\psi_{c}$. Assuming

$$
\langle d\rangle=d\left(-L_{y} / 2\right)=\left(1+d_{\min }\right) / 2
$$

leads to

$$
\frac{\psi_{c}}{\psi_{0}}=\frac{1}{1+\langle d\rangle}=\frac{2}{3+d_{\min }}<\frac{2}{3} .
$$

Figure 9 shows the GCM results for $\psi_{c}$ as the imposed SST asymmetry is $0^{\circ}, 0.01^{\circ}, 0.1^{\circ}, 0.25^{\circ}, 0.5^{\circ}, 1^{\circ}$, or $3^{\circ} \mathrm{C}$. The solid and dashed lines in Fig. 9 are the theoretical lower and upper bounds on $\psi_{c}$, respectively. While there is no discontinuity as the asymmetry changes from zero to small but finite values, the increase in $\psi_{c}$ is extremely rapid. The prediction of the theory is already met with an SST asymmetry of $0.5^{\circ} \mathrm{C}$, which is for all intent and purpose infinitesimal. The deep velocity field shows that in this case, the DWBC entering the SH feeds southwestward flow that almost reaches the southeastern corner (figure not shown)-hence, deep upwelling is almost entirely fed from the north. For even weaker forcing asymmetries, flow fed from the DWBC crossing the equator does not reach the southeastern corner, and $\mathrm{SH}$ deep eastern boundary upwelling is fed partially from the south. Thus, an assumption leading to the derivation of the lower bound is violated, and the lower bound does not apply.

The GCM results are also consistent with the theoretical upper bound (no value above the dashed line in Fig. 9). Likewise in accord with the theory are the numerical results of $\mathrm{KM}$, who with a pole-to-pole temperature difference of $0.6^{\circ} \mathrm{C}$ (the smallest asymmetry used by them) obtained a cross-equatorial transport of about half of the one-hemisphere overturning. In addition, the theoretical upper bound used here is consistent with KM's numerical results; there, in the extreme case of no surface density gradient at all in the $\mathrm{SH}$, cross-hemispheric flow was about $60 \%$ of the strength of the one-hemisphere solution.

Combining Eqs. (8) and (11) allows us to solve for $\psi_{c}$ and $d_{\text {min }}$. Then, Eq. (10) is used to find $\psi_{N}$ and $\psi_{S}$. The theoretical predictions are marked as circles in both panels of Fig. 8. Using $D=350 \mathrm{~m}, H_{r}=2000 \mathrm{~m}, \Delta \rho$ $=0.08 \mathrm{~kg} \mathrm{~m}^{-3}, \psi_{0}=13 \mathrm{~Sv}$, and $f=0.5 \times 10^{-4} \mathrm{~s}^{-1}$, the theory predicts $7.3 \mathrm{~Sv}$ of interhemispheric flow and a maximum deep east-west density difference in the $\mathrm{SH}$ of $0.018 \mathrm{~kg} \mathrm{~m}^{-3}$ at $28^{\circ} \mathrm{S}$. For comparison, the numerical results are, in this order, $7.7 \mathrm{~Sv}, 0.022 \mathrm{~kg} \mathrm{~m}^{-3}$, and $40^{\circ} \mathrm{S}$. Moreover, advective-diffusive balance together with Eqs. (1), (2), and (14) imply that

$$
\frac{\psi_{c}}{\psi_{0}}=\frac{D_{E}^{-1}}{2 D^{-1}}=\frac{2}{3+d_{\min }},
$$

where the 2 in the denominator of the middle term arises because the symmetric overturning upwells both along the eastern and the western boundaries. From Eq. (15), it follows that

$$
D_{E}=D\left(3+d_{\min }\right) / 4 \text {. }
$$

With $d_{\text {min }}=0.5533$ (see Fig. 8), this means that $D_{E}=$ 


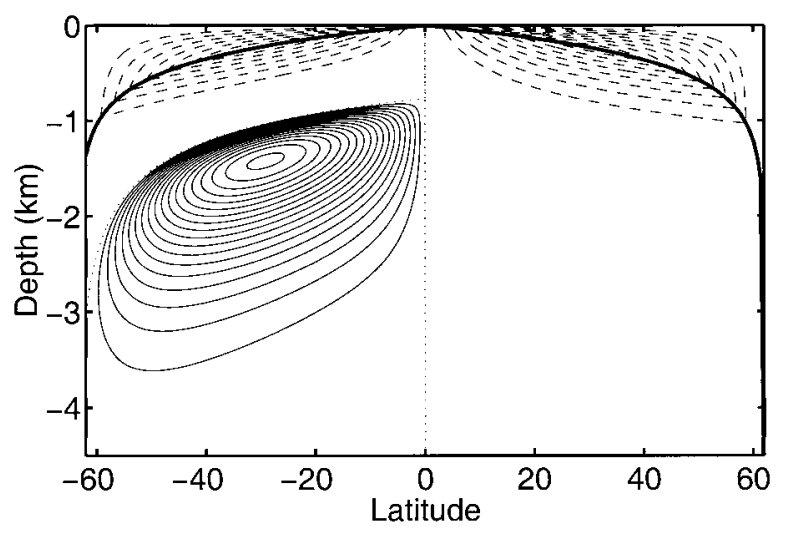

FIG. 10. Theoretical solution for an imposed pole-to-pole surface density difference, $\Delta \rho$, of $0.08 \mathrm{~kg} \mathrm{~m}^{-3}(0.02$ of pole-to-equator density contrast). Shown is the density difference, eastern boundary minus western boundary, according to Eq. (3); thin solid: positive values, contour interval is $0.001 \mathrm{~kg} \mathrm{~m}^{-3}$; dashed: negative values, contour interval is $0.1 \mathrm{~kg} \mathrm{~m}^{-3}$. Heavy solid: (negative) depth of convection, $z_{\rho}$. Dotted: zero line.

$0.89 D$. The eastern boundary thermocline is only slightly sharper in the $\mathrm{SH}$ than in the $\mathrm{NH}$, the theory thus predicting the near-symmetry seen in the GCM (Fig. 3) and anticipated in the discussion of mixed layer depth above. As one consequence, the difference between $\mathrm{SH}$ eastern and western boundary scale heights implies the theoretical prediction of equatorially asymmetric western boundary stratification.

Figure 10 gives the theoretical prediction of the eastwest density difference as a function of latitude and depth, calculated from Eq. (A2) in the appendix and using the scale heights as determined above. Compared to the GCM (Fig. 2), it shows noticeable differences such as the larger gap between negative and positive values in the $\mathrm{SH}$, the lack of extent across the equator, the lack of penetration to the bottom, and the too far equatorward position of the maximum. On the other hand, the general structural commonalities are very encouraging, such as mixed layer depths, the patterns of positive and negative values, the relative symmetry about the equator in the thermocline, and the orders of magnitude. Figures 2 and 10 together indicate that the theory captures the salient features of the GCM's three-dimensional dynamics.

\section{Asymmetric mixing strength}

We now impose a different type of asymmetry, by employing differing vertical mixing coefficients between the NH and SH. Munk and Wunsch (1998) discussed the evidence for the transport of tidal energy across the equator in the Atlantic, which would lead to stronger mixing in the North Atlantic. Moreover, topographic roughness is presumably not uniform over the globe, so vertical mixing would not be uniformly distributed with latitude either. We implement this inhomogeneity in the simplest possible way. One hemisphere has $k_{v}=8 \times 10^{-4} \mathrm{~m}^{2} \mathrm{~s}^{-1}$, while in the other hemisphere $k_{v}=2 \times 10^{-4} \mathrm{~m}^{2} \mathrm{~s}^{-1}$; as before, mixing is zero away from the perimeter. Hence, the averaged mixing coefficient is the same as before. We wish to explore how asymmetries in mixing interact with asymmetries in forcing, so the same asymmetry in restoring SST is used as before (linear latitudinal dependence, with northern boundary SST restored to a value $1^{\circ} \mathrm{C}$ lower than southern boundary SST). Four essentially distinct experiments can be carried out.

1) AS1: High northern density, symmetric mixing (standard experiment, see section 3)

2) AS2: High northern density, high northern mixing

3) AS3: High northern density, high southern mixing

4) AS4: Symmetric density, high southern mixing.

The meridional streamfunction of experiment AS1 is displayed in Fig. 1b, the stream functions from the other three experiments in Fig. 11. If, in addition to the surface density asymmetry, vertical mixing is enhanced in the NH (expt AS2, Fig. 11a), the NH source strength remains almost the same but much of the water upwells in the $\mathrm{NH}$ and is hence short-circuited: only $5.5 \mathrm{~Sv}$ rather than 7.7 Sv cross the equator. This does not confirm the speculation in Munk and Wunsch (1998, p. 1996, their footnote 15) that the high tidal energy dissipation in the North Atlantic might cause the large heat transport convergence there, which is a consequence of large crosshemispheric mass transport (e.g., Macdonald and Wunsch 1996). Rather, it is the high mixing and hence upwelling rate in the lighter hemisphere that leads to large cross-hemispheric mass transport (9.9 Sv, expt AS3, Fig. 11b). Notice that the maximum overturning strength is similar between all these experiments, at just below $18 \mathrm{~Sv}$ in the $\mathrm{NH}$ and around $5 \mathrm{~Sv}$ in the $\mathrm{SH}$. Finally, the run with symmetric forcing but asymmetric diffusivities (expt AS4, Fig. 11c) shows both NH and $\mathrm{SH}$ convection depths and overturning strengths very similar to the symmetrical solution, but the increased mixing in the SH induces greater upwelling, 3.9 Sv of which have to be supplied from the NH. Thus, asymmetric mixing produces cross-equatorial flow, but unlike asymmetric surface density, it does not make one cell extend very far into the other hemisphere.

The theory of section 4 reasonably explains the strength of cross-equatorial flow in experiments AS1AS3. We expect between 0.5 and 0.7 of the symmetric THC strength to cross the equator. According to Eq. (2), the symmetric overturning scales like $k_{v, S}^{2 / 3}$, where the subscript denotes the different mixing in $\mathrm{SH}$ and $\mathrm{NH}$; if we assume that the same fraction of it crosses the equator in each of the experiments, cross-equatorial flow strength should likewise scale like $k_{v, S}^{2 / 3}$. Theoretically, then, one would expect ratios of 1:(1/1.85):1.37 for, in turn, experiments AS1, AS2, and AS3. The numerical runs give ratios of $1:(1 / 1.39): 1.30$, a reasonable agreement. The ratio between the two extreme runs, experiments AS2 and AS3, is 1:2.5 and 1:1.79 for theory and experiment, respectively. 
(a) Strong mixing in denser hemisphere

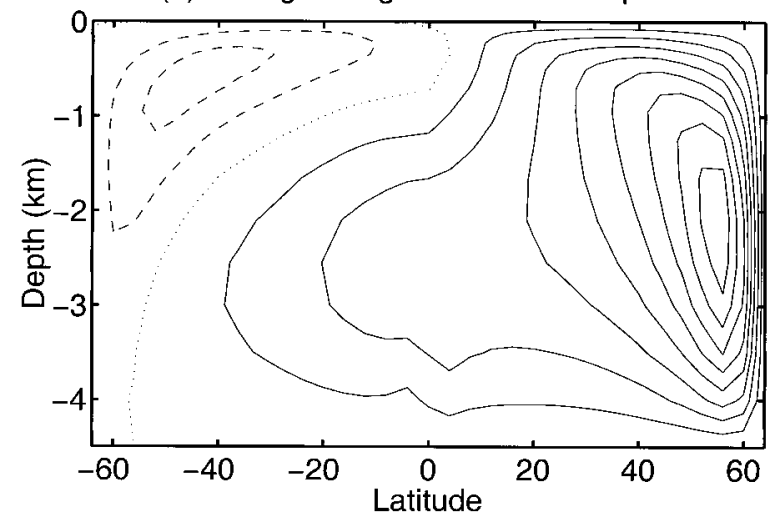

(b) Strong mixing in lighter hemisphere



(c) Asymmetric mixing, symmetric buoyancy



FIG. 11. Meridional overturning: (a) Experiment AS2: high northern density, high northern mixing. (b) Experiment AS3: high northern density, high southern mixing, (c) Experiment AS4: symmetric density, high southern mixing. Contour interval is $2 \mathrm{~Sv}$, negative values are dashed, and the zero contour is dotted. The flow is such that high values are to the right, that is, clockwise around a high and anticlockwise around a low.

\section{Discussion and conclusions}

We have used a two-hemisphere, single-basin idealized GCM driven only by buoyancy forcing to investigate the dynamics of equatorially asymmetric thermohaline circulations under asymmetric surface forcing and mixing intensity. As in KM, an imposed high-latitude surface density asymmetry as small as one percent of the pole-to-equator density contrast is sufficient to cause interhemispheric flow of the same order of magnitude as the total overturning. Geostrophy demands that there be a reversal near the equator in the deep pressure difference between eastern and western boundaries, and across the deep western boundary current (DWBC), which is indeed recognizable as a low-density anomaly in South Atlantic hydrographic sections (e.g., Warren 1981, his Fig. 1.10). Near the surface, however, there is still significant poleward mass transport in the $\mathrm{SH}$, which requires a zonal-mean zonal pressure gradient of the same sign as in the NH. The associated thermocline east-west density difference arises largely because there is upwelling and stable stratification all along the western boundary whereas there is a mixed layer, deepening poleward, along the eastern boundary. Hence, the warm surface water reaches deeper along the eastern boundary at any given latitude, giving the correct sign of thermal wind shear of the zonally integrated meridional flow (M97).

The subthermocline asymmetry arises because SH western boundary density decays towards the abyssal value more slowly with depth than does $\mathrm{SH}$ eastern boundary density. Hence, while western wall density starts higher than eastern wall density near the surface, at some depth the eastern wall density has caught up and a reversal in mean zonal density gradient occurs. Associated is widespread subthermocline downwelling in the SH western boundary region, in contrast to unidirectional upwelling near the $\mathrm{NH}$ western boundary. The DWBC, after crossing the equator, feeds downwelling rather than upwelling; the downwelled water then turns eastward and southeastward to upwell at the eastern wall.

To explain the differing density scale heights near the eastern and western walls in the $\mathrm{SH}$, we have analyzed the spinup from the symmetric to the asymmetric solution. Enhanced convection at high northern latitudes leads to upwelling and positive density anomalies at the western boundary, which are advected southward by the mean DWBC until they reach the equator. The dense anomaly propagates eastward along the equator to the eastern boundary, where it splits and moves both northward and southward. Only then, after approximately 100 years, is significant cross-equatorial transport accomplished. This advective picture shares the propagation pattern of the Kelvin wave dynamics of Kawase (1987), but it occurs two orders of magnitude more slowly.

The steady-state results can be explained by a generalization of the theory of M97. Fundamental assumptions are that the SH western boundary has more diffusive stratification than the SH eastern boundary (supporting the deep southward THC geostrophically), that the DWBC in the SH feeds upwelling purely at the eastern wall, and that the various THC branches are controlled in strength by vertical diffusion. As one con- 
sequence, mixed layer depths and zonally averaged temperature are only slightly asymmetric about the equator, except at very high latitudes.

The theory predicts that cross-equatorial flow is between one half and two thirds of the strength that the $\mathrm{SH}$ could sustain in isolation. The existence of a lower bound arises from the theory because the deep crossequatorial transport must feed strong enough upwelling along the eastern boundary to create a positive deep east - west density difference. In the numerical model, a very sharp transition from the symmetric to an asymmetric solution occurs upon the introduction of even a tiny surface density asymmetry. This explains why the symmetric solution has not been realized in asymmetric geometries (Marotzke and Willebrand 1991) and is hard to obtain under mixed boundary conditions, where salinity advection can easily set up asymmetries in surface density (KM). Our results also point to limitations of box models or two-dimensional models, which often link local flow to the local zonal-mean meridional density gradient (e.g., Welander 1986; Marotzke et al. 1988). These models require a lower near-surface density at the upwelling high latitudes than at the equator, to support cross-hemispheric flow, in contrast to the GCM and the real Atlantic. In a box model, a nonlocal dynamical law appears more appropriate, such as linking the cross-equatorial flow to the pole-to-pole density difference (see Rooth 1982 or its extension by Scott et al. 1999).

The theoretically derived east-west density difference (as a function of latitude and depth) agrees reasonably well with the numerical result, as does the theoretical cross-equatorial overturning. Hence, we feel confident that our theory explains the most salient features of cross-equatorial dynamics. Overall, we think that our assumptions are plausible if not always derivable from first principles; moreover, we present an inherently consistent theoretical picture. We know of no other paper that attempts to explain the force balance (i.e., dynamics) of interhemispheric thermohaline flow together with its thermodynamics.

As in the single-hemisphere solutions, confining diapycnal mixing to the side boundaries does not substantially alter the gross solution properties, as judged by comparison with KM. Knowing the exact longitudinal distribution of mixing appears less important than knowing its overall intensity. On the other hand, the interhemispheric distribution of mixing intensity has a profound impact on the circulation if, in addition, there is asymmetry in surface forcing with respect to the equator. If mixing is enhanced in the hemisphere with the denser, dominant deep-water source and overturning cell, much of this mass transport is short-circuited and wells up in the dominant hemisphere, leading to considerable reduction in cross-equatorial transport. If, however, mixing is enhanced in the lighter hemisphere, the resulting strong upwelling must be fed by vigorous cross-equatorial flow from the dominant hemisphere. If surface forcing is symmetric about the equator, asymmetric mixing leads to considerably weaker asymmetries in circulation.

High-latitude surface salinities and hence densities determine the qualitative global THC pattern, but the mixing distribution sets the strength of the overturning cells in the different ocean basins, if our results can be generalized to the World Ocean. This implies that the overall amount of mixing limits the global integral of thermohaline overturning (Munk 1966). Wunsch (1998) has shown that the main work done by the wind on the ocean occurs in the Southern Ocean; if one assumes that this locally leads to enhanced mixing, our results suggest that the vigorous deep interhemispheric flow in the Atlantic owes as much to mixing in the Southern Ocean as to the high North Atlantic salinity. Moreover, one may speculate that the numerical result of Southern Ocean wind impact on Atlantic overturning (Toggweiler and Samuels 1993, 1998) arises because of two different mixing processes in the Antarctic Circumpolar Current. Stronger winds lead to stronger Ekman-driven upwelling and stronger entrainment (hence mixing) into the mixed layer. They also cause stronger baroclinicity, hence stronger horizontal, diapycnal mixing, leading to stronger overall and cross-equatorial flow. The choice of eddy parameterization is critical in this latter connection, as discussed extensively by Kamenkovich et al. (2000).

Acknowledgments. We thank Alistair Adcroft, Mick Follows, John Marshall, Jeff Scott, and Carl Wunsch for valuable comments on earlier versions of this manuscript. Comments by two reviewers led to considerable improvement. JM was supported by the Tokyo Electric Power Company through the TEPCO/MIT Environmental Research Program, by NSF Grant OCE9801800 , and to $40 \%$ (\$6000) jointly by the Northeast Regional Center of the National Institute for Global Environmental Change and by the Program for Computer Hardware, Applied Mathematics, and Model Physics (both with funding from the U.S. Department of Energy). BAK was supported by NSF Grant OCE9521138.

\section{APPENDIX}

\section{Derivation of Eq. (5)}

In the main text [Eq. (3)], it was stated for the SH density structure that

$$
\rho_{W}(y, z)=\rho_{P}+\left[\rho_{S}(y)-\rho_{P}\right] \exp \left[z / D_{W}(y)\right],
$$

ML:

$$
\rho_{E}(y, z)=\rho_{S}(y),
$$

below ML:

$$
\rho_{E}(y, z)=\rho_{P}+\left(\rho_{T}-\rho_{P}\right) \exp \left(z / D_{E}\right) .
$$


The subscripts $E$ and $W$ stand for eastern and western boundary, respectively, and the mixed layer (ML) is defined as $z>z_{\rho}(y)$. Here, $z_{\rho}(y)$ is given by Eq. (4), which can be viewed as the matching condition ensuring continuity (and static stability) along the eastern boundary. The density difference between eastern and western walls follows as

ML:

$$
\begin{aligned}
& \rho_{E}(y, z)-\rho_{W}(y, z) \\
& \quad=\left[\rho_{S}(y)-\rho_{P}\right]\left\{1-\exp \left[z / D_{W}(y)\right]\right\}
\end{aligned}
$$

below ML:

$$
\begin{aligned}
& \rho_{E}(y, z)-\rho_{W}(y, z) \\
& \quad=\left(\rho_{T}-\rho_{P}\right) \exp \left(z / D_{E}\right)-\left[\rho_{S}(y)-\rho_{P}\right] \exp \left[z / D_{W}(y)\right] .
\end{aligned}
$$

At $z=z_{\rho}(y)$, the two expressions (A2a) and (A2b) match, meaning that the east is less dense than the west - the first term on the right-hand side of Eq. (A2b) dominates. But if $D_{E}<D_{W}(y) \ll H$, where $H$ is total depth, the eastern boundary approaches its abyssal density faster with depth than does the western boundary, and the east-west density difference reverses at some finite depth above the bottom. The depth $\left|z_{0}\right|$ at which this occurs is defined by

$$
\begin{aligned}
& \left(\rho_{T}-\rho_{P}\right) \exp \left(z_{0} / D_{E}\right) \\
& \quad \equiv\left[\rho_{S}(y)-\rho_{P}\right] \exp \left[z_{0} / D_{W}(y)\right],
\end{aligned}
$$

from which it follows that

$$
\begin{aligned}
z_{0}(y) & =\frac{1}{1-d(y)} D_{E} \ln \left[\frac{\rho_{P}-\rho_{S}(y)}{\rho_{P}-\rho_{T}}\right] \\
& =\frac{1}{1-d(y)} z_{\rho}(y),
\end{aligned}
$$

where $d(y) \equiv D_{E} / D_{W}(y)$ is the ratio of the thermocline scale heights in the east and west of the SH. Notice that the argument of the logarithm is less than one. It follows that for $D_{E}<D_{W}(y)$, that is, $d(y)<1$, the density reversal occurs at some finite, negative height, with $\left|z_{0}(y)\right|>\left|z_{\rho}(y)\right|$. If $D_{E}>D_{W}(y)$, that is, $d(y)>1, z_{0}$ is positive and no density crossover occurs. Moreover, if $D_{W}(y)$ is only slightly larger than $D_{E}, d(y)$ is close to unity and $\left|z_{0}\right|$ is close to $H$.

The vertical coordinate $z_{\max }$ of the maximum positive east-west density difference can be obtained from setting to zero the vertical derivative of Eq. (A2b), which yields

$$
\begin{aligned}
z_{\max }(y) & =\frac{1}{1-d(y)} D_{E} \ln \left[d(y) \frac{\rho_{P}-\rho_{S}(y)}{\rho_{P}-\rho_{T}}\right] \\
& =\frac{1}{1-d(y)}\left\{z_{\rho}(y)+D_{E} \ln [d(y)]\right\} .
\end{aligned}
$$

Inserting Eq. (A5) into Eq. (A2b) gives the maximum east-west density difference in the $\mathrm{SH}$ as

$$
\begin{aligned}
\Delta \rho_{\max }(y) \equiv & \rho_{E}\left(y, z_{\max }\right)-\rho_{W}\left(y, z_{\max }\right) \\
= & \left(\rho_{T}-\rho_{P}\right)\left[d(y) \frac{\rho_{P}-\rho_{S}(y)}{\rho_{P}-\rho_{T}}\right]^{1 /(1-d(y))} \\
& -\left[\rho_{S}(y)-\rho_{P}\right]\left[d(y) \frac{\rho_{P}-\rho_{S}(y)}{\rho_{P}-\rho_{T}}\right]^{d(y) /(1-d(y))}
\end{aligned}
$$

The terms in Eq. (A6) can be combined to give

$$
\begin{aligned}
\Delta \rho_{\max }(y)= & \left(\rho_{P}-\rho_{T}\right)\left(\frac{\rho_{P}-\rho_{S}(y)}{\rho_{P}-\rho_{T}}\right)^{1 /(1-d(y))} d(y)^{d(y) /(1-d(y))} \\
& \times(1-d(y)),
\end{aligned}
$$

which is Eq. (5) in the main text.

\section{REFERENCES}

Armi, L., 1978: Some evidence for boundary mixing in the deep ocean. J. Geophys. Res., 83, 1971-1979.

Bryan, F., 1986: High-latitude salinity effects and interhemispheric thermohaline circulations. Nature, 323, 301-304.

, 1987: Parameter sensitivity of primitive equation ocean general circulation models. J. Phys. Oceanogr., 17, 970-985.

Bryan, K., 1969: A numerical method for the study of the circulation of the world ocean. J. Comput. Phys., 4, 347-376.

_- 1984: Accelerating the convergence to equilibrium of ocean climate models. J. Phys. Oceanogr., 14, 666-673.

Colin de Verdière, A., 1988: Buoyancy driven planetary flows. $J$. Mar. Res., 46, 215-265.

Cox, M. D., 1984: A primitive equation, three-dimensional model of the ocean. GFDL Ocean Group Tech. Rep. No. 1, GFDL/Princeton University, 143 pp. [Available from GFDL/NOAA, Princeton University, P.O. Box 308, Princeton, NJ 08542.]

_ 1989: An idealized model of the World Ocean. Part I: The global-scale water masses. J. Phys. Oceanogr., 19, 1730-1752.

Döscher, R., C. W. Böning, and P. Herrmann, 1994: Response of circulation and heat transport in the North Atlantic to changes in thermohaline forcing in northern latitudes: A model study. $J$. Phys. Oceanogr., 24, 2306-2320.

England, M. H., 1993: Representing the global-scale water masses in ocean general circulation models. J. Phys. Oceanogr., 23, $1523-1552$.

Gerdes, R., and C. Köberle, 1995: On the influence of DSOW in a numerical model of the North Atlantic general circulation. $J$. Phys. Oceanogr., 25, 2624-2642.

Gordon, A. L., 1986: Interocean exchange of thermocline water. $J$. Geophys. Res., 91, 5037-5046.

Hsieh, W. W., M. K. Davey, and R. C. Wajsowicz, 1983: The free Kelvin wave in finite-difference numerical models. J. Phys. Oceanogr., 13, 1383-1397.

Huang, R. X., and R. L. Chou, 1994: Parameter sensitivity of the saline circulation. Climate Dyn., 9, 391-409.

Hughes, T. C. M., and A. J. Weaver, 1994: Multiple equilibria of an asymmetric two-basin ocean model. J. Phys. Oceanogr., 24, 619-637.

Kamenkovich, I., J. Marotzke, and P. H. Stone, 2000: Factors affecting heat transport in an ocean general circulation model. J. Phys. Oceanogr., 30, 175-194.

Kawase, M., 1987: Establishment of deep ocean circulation driven by deep-water production. J. Phys. Oceanogr., 17, 2294-2317. 
Killworth, P. D., 1985: A two-level wind and buoyancy driven thermocline model. J. Phys. Oceanogr., 15, 1414-1432.

Klinger, B. A., and J. Marotzke, 1999: Behavior of double hemisphere thermohaline flows in a single basin. J. Phys. Oceanogr., 29, 382-399.

Ledwell, J. R., and A. Bratkovich, 1995: A tracer study of mixing in the Santa Cruz Basin. J. Geophys. Res., 100, 20 681-20 704.

Macdonald, A. M., and C. Wunsch, 1996: An estimate of global ocean circulation and heat fluxes. Nature, 382, 436-439.

Manabe, S., and R. J. Stouffer, 1988: Two stable equilibria of a coupled ocean-atmosphere model. J. Climate, 1, 841-866.

Marotzke, J., 1997: Boundary mixing and the dynamics of threedimensional thermohaline circulations. J. Phys. Oceanogr., 27, $1713-1728$

—, and J. Willebrand, 1991: Multiple equilibria of the global thermohaline circulation. J. Phys. Oceanogr., 21, 1372-1385.

- , and J. R. Scott, 1999: Convective mixing and the thermohaline circulation. J. Phys. Oceanogr., 29, 2962-2970.

-, P. Welander, and J. Willebrand, 1988: Instability and multiple steady states in a meridional-plane model of the thermohaline circulation. Tellus, 40A, 162-172.

McDermott, D. A., 1996: The regulation of northern overturning by Southern Hemisphere winds. J. Phys. Oceanogr., 26, 1234 1255 .

Mikolajewicz, U., E. Maier-Reimer, T. J. Crowley, and K.-Y. Kim, 1993: Effect of Drake and Panamanian gateways on the circulation of an ocean model. Paleoceanography, 8, 409-426.

Munk, W., 1966: Abyssal recipes. Deep-Sea Res., 13, 707-730.

—, and C. Wunsch, 1998: Abyssal recipes II: energetics of tidal and wind mixing Deep-Sea Res., 45, 1977-2010.

Polzin, K. L., J. M. Toole, J. R. Ledwell, and R. W. Schmitt, 1997: Spatial variability of turbulent mixing in the abyssal ocean. Science, 276, 93-96.

Rahmstorf, S., 1996: On the freshwater forcing and transport of the Atlantic thermohaline circulation. Climate Dyn., 12, 799-811.

Rooth, C., 1982: Hydrology and ocean circulation. Progress in Oceanography, Vol. 11. Pergamon, 131-149.

Scott, J. R., J. Marotzke, and P. H. Stone, 1999: Interhemispheric thermohaline circulation in a coupled box model. J. Phys. Oceanogr., 29, 351-365.

Suginohara, N., and M. Fukasawa, 1988: Set-up of deep circulation in multi-level numerical models. J. Oceanogr. Soc. Japan, 44, 315-336.

Toggweiler, J. R., and B. Samuels, 1993: Is the magnitude of the deep outflow from the Atlantic Ocean actually governed by Southern Hemisphere winds? The Global Carbon Cycle, M. Heimann, Ed., Springer, 303-331.

- and _ 1998: On the ocean's large-scale circulation near the limit of no vertical mixing. J. Phys. Oceanogr., 28, 1832-1852.

Tziperman, E., 1997: Inherently unstable climate behavior due to weak thermohaline ocean circulation. Nature, 386, 592-595.

Wajsowicz, R. C., 1986: Adjustment of the ocean under buoyancy forces. Part II: The role of planetary waves. J. Phys. Oceanogr., 16, 2115-2136.

- and A. E. Gill, 1986: Adjustment of the ocean under buoyancy forces. Part I: The role of Kelvin waves. J. Phys. Oceanogr., 16, 2097-2114

Wang, X., P. H. Stone, and J. Marotzke, 1999: Global thermohaline circulation, Part I: Sensitivity to atmospheric moisture transport. J. Climate, 12, 71-82.

Warren, B. A., 1981: Deep circulation of the World Ocean. Evolution of Physical Oceanography, Scientific Surveys in Honor of Henry Stommel, B. A. Warren and C. Wunsch, Eds., The MIT Press, $6-41$.

Welander, P., 1986: Thermohaline effects in the ocean circulation and related simple models. Large-Scale Transport Processes in Oceans and Atmosphere, J. Willebrand and D.L.T. Anderson, Eds., NATO ASI Series, D. Reidel, 163-200.

Winton, M., 1996: The role of horizontal boundaries in parameter sensitivity and decadal-scale variability of coarse-resolution ocean general circulation models. J. Phys. Oceanogr., 26, 289304.

Wunsch, C., 1970: On oceanic boundary mixing. Deep-Sea Res., 17, 293-301.

, 1998: The work done by the wind on the ocean general circulation. J. Phys. Oceanogr., 28, 2332-2340.

Zhang, J., R. W. Schmitt, and R. X. Huang, 1999: The relative influnce of diapycnal mixing and hydrologic forcing on the stability of the thermohaline circulation. J. Phys. Oceanogr., 29, 1096-1108. 